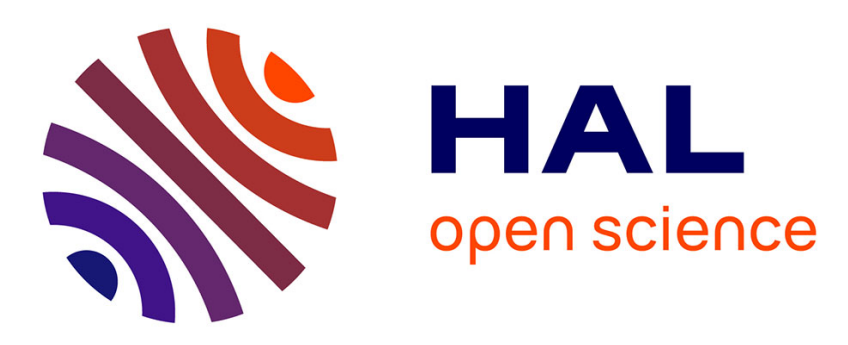

\title{
Impact of Hybrid Pansharpening Approaches Applied to Hyperspectral Images
}

\author{
Giorgio Licciardi, Miguel Angel Veganzones, G Vivone, Laëtitia Loncan, \\ Jocelyn Chanussot
}

\section{- To cite this version:}

Giorgio Licciardi, Miguel Angel Veganzones, G Vivone, Laëtitia Loncan, Jocelyn Chanussot. Impact of Hybrid Pansharpening Approaches Applied to Hyperspectral Images. WHISPERS 2015 - 7th Workshop on Hyperspectral Image and Signal Processing: Evolution in Remote Sensing, Jun 2015, Tokyo, Japan. 10.1109/WHISPERS.2015.8075402 · hal-01121191

\section{HAL Id: hal-01121191 \\ https://hal.science/hal-01121191}

Submitted on 27 Feb 2015

HAL is a multi-disciplinary open access archive for the deposit and dissemination of scientific research documents, whether they are published or not. The documents may come from teaching and research institutions in France or abroad, or from public or private research centers.
L'archive ouverte pluridisciplinaire HAL, est destinée au dépôt et à la diffusion de documents scientifiques de niveau recherche, publiés ou non, émanant des établissements d'enseignement et de recherche français ou étrangers, des laboratoires publics ou privés. 


\title{
IMPACT OF HYBRID PANSHARPENING APPROACHES APPLIED TO HYPERSPECTRAL IMAGES
}

\author{
G. Licciardi ${ }^{1}$, M.A. Veganzones ${ }^{1}$, G. Vivone ${ }^{2}$, L. Loncan $^{1,3}$, J. Chanussot $^{1,4}$. \\ 1 GIPSA-lab, Grenoble-INP, Saint Martin d'Hères, France \\ 2 Universita degli Studi Salerno, Italy \\ 3 ONERA, France \\ 4 Faculty of Electrical and Computer Engineering, University of Iceland, Reykjavik, Iceland
}

\begin{abstract}
Pansharpening techniques can be divided into component substitution (CS) and multi-resolution analysis (MRA) based methods. Generally, the CS methods result in fused images having high spatial quality but the fused images suffer from spectral distortions. On the other hand, images obtained using MRA techniques are not as sharp as CS methods but they are spectrally consistent. Both substitution and filtering approaches are considered adequate when applied to multispectral and PAN images, but have many drawbacks when the low-resolution image is a hyperspectral image. Based on these findings, the use of a hybrid approach, combining the better spatial information of $\mathrm{CS}$ and the more accurate spectral information of MRA techniques, may result in an improvement in terms of spectral quality, spatial sharpness as well as computational time.
\end{abstract}

Index Terms - Pansharpening, Hyperspectral, multiresolution analysis, component substitution

\section{INTRODUCTION}

Generally, for satellite images, multispectral (MS) and hyperspectral (HS) images cover a wide spectral range with moderate to high spectral resolution, but with a low spatial resolution if compared with a panchromatic (PAN) image. However the drawback of the PAN image is that it has no spectral information beyond that which is averaged within the bandpass of the PAN image. Thus, for many application it is desirable to have images having both high spectral and spatial resolutions.

Pansharpening, or image fusion, is the process of improving the spatial quality of a low spatial resolution image by fusing it with a high resolution PAN image [1] [2]. One of the main challenges in pansharpening is to improve the spatial resolution while preserving the original spectral information. In the literature, several methods have been proposed for pansharpening of MS and PAN images and generally fall into component substitution (CS) and multi-resolution analysis (MRA) methods.
CS-based techniques have the main advantages of a high spatial sharpness of the enhanced image, a fast and easy implementation, and robustness to misregistration errors and aliasing [3]. On the other hand, MRA methods have the relevant advantage to preserve the spectral information of the original image. However, when these approaches are considered adequate if applied to MS and PAN images, they may have several drawbacks, in terms of spectral distortion and increase of computational time, when the low-resolution image is a HS image. In particular, due to the different spectral coverage between the PAN and the HS images, CS methods result in enhanced images affected by strong spectral distortions. In general, these approaches are considered adequate if applied to MS and PAN images, they may have several drawbacks, in terms of spectral distortion and increase of computational time, when the low-resolution image is a HS image. In particular, CS methods present a fast and easy implementation and permit to obtain enhanced images having high spatial quality. However, due to the substitution process, CS methods usually introduce spectral distortions [4]. On the other hand, the images obtained using MRA approaches are not usually as sharp as the ones obtained following CS methods but are spectrally consistent with the original images [4]. From this point of view, one of the main challenges for fusing low-resolution HS and high-resolution PAN data is to find an appropriate balance between spectral and spatial preservation. A possible solution to overcome mutual limitations of both CS and MRA approaches, is to use hybrid approaches, combining the different classes of methods in order to find an appropriate balance between spectral and spatial preservation. Aim of this paper is to evaluate the effect of using a hybrid framework in terms of quality of the enhanced image as well as in computational time.

\section{HYPERSPECTRAL PANSHARPENING}

Many of the methods proposed in the literature are considered adequate when applied to multispectral images, but have many drawbacks when the low-resolution image is a hyper- 
spectral image. This means that the best method to improve the spatial quality of a hyperspectral image avoiding spectral distortions vary case by case.

Among the component substitution techniques, PCA pansharpening approach [5] [6] is based on the Principal Component Analysis orthogonal transformation of the low resolution image, where the first principal component is projected in the direction of the highest variance possible. The amount of the data variance is the greatest in the first component, and decreases in the following ones. Thus, if the variables of the input data (i.e. the HS bands) are highly correlated, the first PC collects the information that is common to all bands used as input data to the PCA, corresponding to the spatial information, while the spectral information that is specific to each band is captured in the other principal components. Assuming that the variables of the input data are highly correlated, the first PC will correspond to the average of all the input variables, that, in turn, is very similar to a PAN image. In this way is possible to introduce spatial information in the low resolution image by substituting the first PC with the PAN image. It is clear that the spectral distortion of the enhanced image is directly linked to the spectral coverage of the HS and PAN images. However, other source of spectral distortion may be caused by the way the first PC is obtained from the bands of the HS image. From this point of view, the spectral quality can be improved by using nonlinear transformations, such as the Nonlinear Principal Component Analysis (NLPCA) [7]. Basically, MRA methods for are based on a spatial filter applied to the PAN image in order to produce spatial details to be injected in the HS image. The main difference between MRA methods is on the transformations used for the detail extraction and their injection.

The use of pyramidal decomposition as an efficient image representation tool was proposed in [8] by performing a decomposition of the original image via successive lowpass Gaussian filtering and decimation processes. The differences between consecutive levels of a Gaussian pyramid define the so-called Laplacian pyramid. This method was found to be especially suitable for hyperspectral pansharpening when an adequate knowledge of the sensor Modulation Transfer Function (MTF) is available [9].

The Smoothing Filter-based Intensity Modulation (SFIM) technique is based on a single linear time-invariant (LTI) low pass filter (LPF) applied to the PAN image [10]. Then the spatial details are obtained from the difference between the original PAN and the filtered images.

In the general framework of hybrid pansharpening, the HS image is firstly projected into a feature space by means of a linear or nonlinear transform, as in CS methods. Then, according to a MRA approach, spatial filters are applied to the PAN image in order to extract spatial details that will be injected in one or more features obtained in the previous step. Finally an inverse transform is applied in order to obtain the

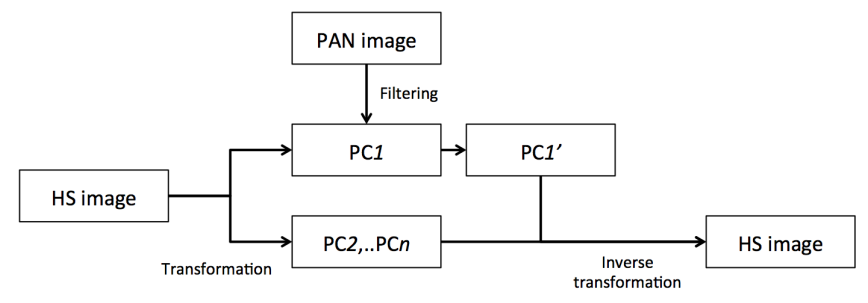

Fig. 1. General scheme for the hybrid pansharpening framework.

enhanced image, as reported in Fig. 1. In this way it is possible to reduce the spectral distortion produced by the substitution process. On the other hand, the spatial enhanced image may present less spatial details than the image obtained using a classic CS technique. Thus, depending on the selected filtering method, the final image may result in an optimal tradeoff between spectral and spatial quality. Few examples of hybrid pansharpening approaches have been proposed in the literature. A hybrid approach has been proposed in [11], where GS and gaussian filters have been combined to enhance the quality of MS images. In [7] the NLPCA transformation has been used for the projection of the HS image in the feature space while the Indusion approach has been used for the injection of the spatula details in one of the nonlinear principal components.

These first approaches demonstrated that a hybrid approach can lead to an effective improvement of the pansharpened image. However, an accurate selection of the pansharpening method is essential to obtain the best tradeoff between spectral quality and spatial consistency, as well as a strong reduction in the computational time.

\section{EXPERIMENTS}

In this section, the proposed framework has been applied to two real datasets. The enhanced images obtained in both experiments are quantitatively evaluated by means of Relative Dimensional Global Error (ERGAS), Spectral Angle Mapper (SAM), Universal Image (UIQI) quality indexes. ERGAS, SAM and UIQI will produce positive values with an ideal value of 0,0 and 1, respectively. However, values that are around 3 are referred to a good image enhancement. SAM is a useful measure of the spectral quality introduced by the fusion process, while ERGAS measure both spectral and spatial quality. A qualitative analysis, through visual inspection, will also be discussed. In particular, we selected PCA and NLPCA for the transformation phase representing linear and nonlinear transformations, respectively. As for the injection phase, the following techniques have been selected:

- MTF GLP Modulation Transfer Function - Generalized Laplacian Pyramid (MTF-GLP) with High Pass Modulation (HPM) injection model algorithm; 
- SFIM Smoothing Filter-based Intensity Modulation (SFIM) algorithm.

It worth to be noticed that, since the used images are real data, a reference image is generally not available for real-life HS image, and a quality analysis through the Wald protocol cannot be carried out. In order to further assess the performances of the proposed method, the results obtained with the hybrid approaches have been compared with those obtained using classical pansharpening techniques applied directly to the HS images.

\subsection{Hyperion-ALI}

On a first experiment the enhanced images have been obtained by fusing Hyperion and ALI images, both carried by the EO-1 satellite and acquired simultaneously over the city of Paris. Hyperion is a grating imaging spectrometer providing 242 hyperspectral bands (from 0.4 to $2.5 \mu \mathrm{m}$ ) with a 30 meter spatial resolution. The ALI PAN instrument provides a panchromatic image covering the visible range from 0.48 to $0.69 \mu \mathrm{m}$ with 10 -meter resolution. Since the two sensors are carried by the same satellite, and the images are acquired simultaneously, the fused image will not be affected by differences in terms of angle of view, atmospherical path, illumination as well as misregistration. Following the proposed hybrid framework, the Hyperion image has been projected in the feature space according to the PCA and NLPCA approaches, respectively. However, differently from the linear PCA, in the NLPCA the components are not ranked in terms of variance. For this reason we injected the spatial details in the component presenting the highest correlation with the PAN image. The, the selected components have been fused with the PAN image according to the SFIM and MTF GLP approaches, respectively. Finally, the enhanced components have been substituted to the original ones and the new feature sets have been reprojected back to the original data space. Table 2 shows the ERGAS, SAM and UIQI quality indexes associated to the different pansharpening techniques. As expected, the CS methods presents the best results in terms of spatial sharpness while MRA techniques preserve the spectral characteristics of the original image. On the other hand, the hybrid approaches present a good tradeoff between spectral and spatial quality. In particular, the PCA-SFIM technique presents the best quality indexes among the hybrid approaches. This can be also noticed by analyzing the images reported in Fig. 2

\subsection{Hyperion-ASTER}

On a second experiment data fusion was applied to Hyperion and ASTER images taken over San Francisco on 31 July, 2002. ASTER sensor provides images in 14 spectral bands (from 0.52 to $11.65 \mu \mathrm{m}$ ) with spatial resolutions between 15 to 90 meters. However, due to the differences in

\begin{tabular}{lccc}
\hline & SAM & ERGAS & UIQI \\
\hline CS methods & & & \\
\hline NLPCA-SUB & 7.6501 & 6.9801 & 0.7947 \\
PCA-SUB & 6.5668 & 5.7291 & 0.7591 \\
\hline MRA methods & & & \\
\hline MTF-GLP & 1.5363 & 3.8105 & 0.6333 \\
SFIM & 1,2800 & 2.8510 & 0.5619 \\
\hline Hybrid methods & & & \\
\hline NLPCA-MTF-GLP & 4.8534 & 4.0677 & 0.7263 \\
NLPCA-SFIM & 4.8122 & 4.9336 & 0.5581 \\
\hline PCA-MTF-GLP & 4.0725 & 4.6327 & 0.5365 \\
PCA-SFIM & 4.7577 & 4.9251 & 0.7325 \\
\hline
\end{tabular}

Table 1. SAM, ERGAS and UIQI quality index values, obtained with the different fusion approaches applied to the Hyperion-ALI dataset.
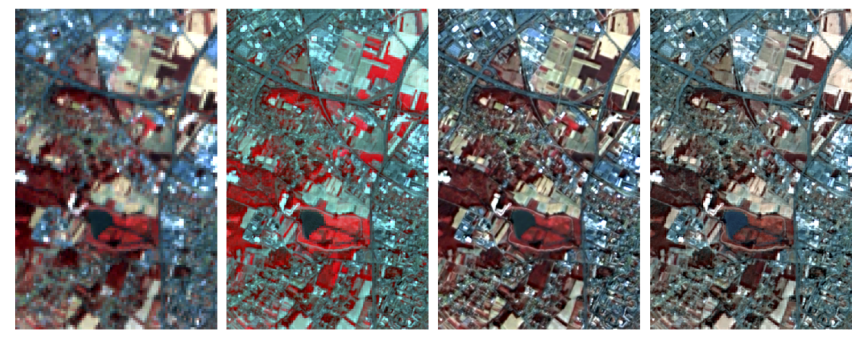

Fig. 2. (From left to right) Original Hyperion image and enhanced images obtained using SFIM, NLPCA-MTF-GLP and PCA-MTFGLP.

\begin{tabular}{lccc}
\hline & SAM & ERGAS & UIQI \\
\hline CS methods & & & \\
\hline NLPCA-SUB & 8.9398 & 6.9545 & 0.9794 \\
PCA-SUB & 4.1264 & 4.9359 & 0.8954 \\
\hline MRA methods & & & \\
\hline MTF-GLP & 1.1062 & 3.2999 & 0.8297 \\
SFIM & 0.7857 & 2.5573 & 0.8614 \\
\hline Hybrid methods & & & \\
\hline NLPCA-MTF-GLP & 8.6369 & 6.9294 & 0.8215 \\
NLPCA-SFIM & 7.8070 & 6.5505 & 0.8432 \\
\hline PCA-MTF-GLP & 3.2088 & 3.3990 & 0.7593 \\
PCA-SFIM & 3.3808 & 3.6832 & 0.7230 \\
\hline
\end{tabular}

Table 2. SAM, ERGAS and UIQI quality index values, obtained with the different fusion approaches applied to the Hyperion-ASTER dataset. 

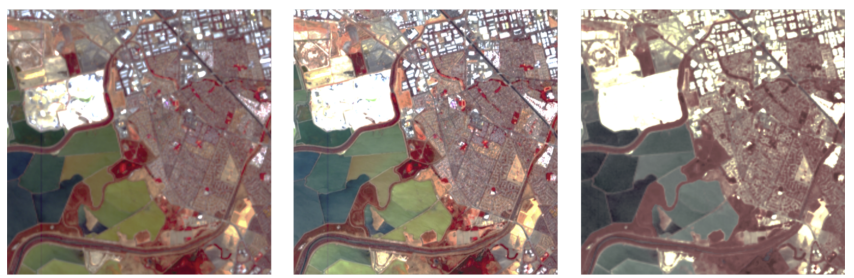

Fig. 3. (From left to right) Original Hyperion image and enhanced images obtained using NLPCA-SFIM and PCA-SFIM.

spectral coverages, only the first three bands of ASTER ( 0.52 to $0.860 ? \mathrm{~m}$ ), with $15 \mathrm{~m}$ ground sampling distance, were averaged to produce a synthetic PAN image. Moreover, we used a Hyperion/VNIR dataset having $30 \mathrm{~m}$ ground sampling distance and 50 bands $(0.43$ to $0.92 \mu \mathrm{m})$. As for the previous experiment, the low resolution image has been projected in the feature space according to the PCA and NLPCA techniques, and the selected components fused with the PAN image according to the SFIM and MTF GLP approaches, respectively. The enhanced components were then reprojected back to the original data space to obtain the fused images. Similarly to the previous experiment MRA methods present the best spectral quality while the sharpest images are obtained using CS methods. As reported in Table 1, the images obtained with the hybrid techniques present an optimal tradeoff between the spectral and spatial quality of the enhanced image. Is important to stress that one of the most important characteristic of NLPCA is the ability of removing noise from hyperspectral images. This explains the high spectral difference between the original image and those obtained with the use of NLPCA.

\section{CONCLUSIONS}

In this paper the synergic use of CS and MRA techniques has been investigated in the framework of hybrid pansharpening. In particular, in the proposed approach a hyperspectral image is projected into a feature space by means of PCA or NLPCA techniques. The obtained components are then enhanced spatially according to a MRA method and reprojected back to the original data space. Several pansharpening techniques have been considered. SAM, ERGAS and UIQI indexes have been used to evaluate the enhanced images in terms of spectral distortion and spatial consistency. Qualitative analysis has been carried out by visual inspecting the enhanced images. The obtained results demonstrate that the use of a hybrid approach, overcoming the limitations of the single techniques, offers a good tradeoff between spectral and spatial quality of the enhanced image.

\section{REFERENCES}

[1] L. Wald, "Data fusion. definitions and architectures - fusion of images of different spatial resolutions," Presses de l'Ecole, Ecole des Mines de Paris, Paris, France, 2002.

[2] F. Palsson, J. R. Sveinsson, J.A. Benediktsson, and H. Aanaes, "Classification of pansharpened urban satellite images," IEEE Journal of Selected Topics in Applied Earth Observations and Remote Sensing., vol. 5, pp. 281-297, 2012.

[3] S. Baronti, B. Aiazzi, M. Selva, A. Garzelli, and L. Alparone, "A theoretical analysis of the effects of aliasing and misregistration on pansharpened imagery," IEEE J. Sel. Topics Signal Process, vol. 5, no. 3, pp. 446-453, 2011.

[4] P. S. Chavez, S. C. Sides, and J. A. Anderson, "Comparison of three different methods to merge multiresolution and multispectral data: Landsat tm and spot panchromatic," Photogramm. Eng. Remote Sens., vol. 57, no. 3, pp. 295-303, 1991.

[5] T. M. Tu, S. C. Su, H. C. Shyu, and P. S. Huang, "A new look at ihs-like image fusion methods," Information Fusion, vol. 2, no. 3, pp. 177 - 186, 2001.

[6] Amro I., Mateos J., Vega M., Molina R., and Katsaggelos A.K., "A survey of classical methods and new trends in pansharpening of multispectral images," EURASIP Journal on Advances in Signal Processing, , no. 79, pp. 1-22, Sep 2011.

[7] G. Licciardi, M. M. Khan, J. Chanussot, A. Montanvert, L. Condat, and C. Jutten, "Fusion of hyperspectral and panchromatic images using multiresolution analysis and nonlinear pca band reduction," EURASIP Journal of Advances in Signal Processing, vol. 2012:207, 2012.

[8] Burt P. and Adelson E., "The laplacian pyramid as a compact image code," IEEE Trans. Commun., vol. 31, no. 4, pp. 532-540, Apr 1983.

[9] Aiazzi B., Alparone L., S. Baronti, A. Garzelli, and Selva M., "MTF-tailored multiscale fusion of highresolution MS and Pan imagery," Photogramm. Eng. Remote Sens., vol. 72, no. 5, pp. 591-596, May 2006.

[10] J. G. Liu, "Smoothing filter based intensity modulation: a spectral preserve image fusion technique for improving spatial details," Int. J. Remote Sens., vol. 21, no. 18, pp. 3461-3472, 2000.

[11] B. Aiazzi, L. Alparone, S. Baronti, and A. Garzelli, "Context-driven fusion of high spatial and spectral resolution images based on oversampled multiresolution analysis," IEEE Trans. Geosci. Remote Sens., vol. 40, pp. 2300-2312, 2002. 